\title{
Applications of Fair Testing
}

E. Brinksma

Department of Computer Science, University of Twente

Postbus 217, NL-7500AE Enschede,brinksma@cs.utwente.nl

A. Rensink

Institut für Informatik, University of Hildesheim ${ }^{1}$

Postf. 101363, D-31113 Hildesheim, rensink@informatik.uni-hildesheim.de

W. Vogler

Institut für Informatik, University of Augsburg

D-86135 Augsburg,vogler@uni-augsburg.de

\section{In: R. Gotzhein and J. Bredereke (Eds.), Formal Description Techniques IX: Theory, Applications and Tools, Chapman \& Hall, 1996}

\begin{abstract}
In this paper we present the application of the fair testing pre-order, introduced in a previous paper, to the specification and analysis of distributed systems. This pre-order combines some features of the standard testing pre-orders, viz. the possibility to refine a specification by the resolution of nondeterminism, with a powerful feature of standard observation congruence, viz. the fair abstraction from divergences. Moreover, it is a precongruence with respect to all standard process-algebraic combinators, thus allowing for the standard algebraic proof techniques by substitution and rewriting. In this paper we will demonstrate advantages of the fair testing pre-order by the application to a number of examples, including a scheduling problem, a version of the Alternating Bit-protocol, and fair communication channels.
\end{abstract}

\section{Keywords}

FDT-application; verification, validation and testing; process algebras; fairness.

\section{INTRODUCTION}

In the past decade, the specification and analysis of distributed systems by means of process-algebraic languages and theories has become an established field of (applied) research. Standardised specification formalisms like LOTOS (Bolognesi and Brinksma (1987), ISO (1989)) as well as basic process algebraic theories like CCS (Milner (1989)), CSP (Hoare (1985)) and ACP (Baeten and Weijland (1990)) are being routinely applied distributed systems in general, and protocol systems in particular.

One of the most interesting features of process algebra is the equational theory of observational behaviour. The developments have shown that there does not really exist one canonical notion of observable behaviour, but that depending on the formalisation of observability, many different notions of observational equivalence (or inclusion) arise (the reader may consult Van Glabbeek (1993) for an overview). Two important families

${ }^{1}$ Partially supported by the HCM network "EXPRESS". 


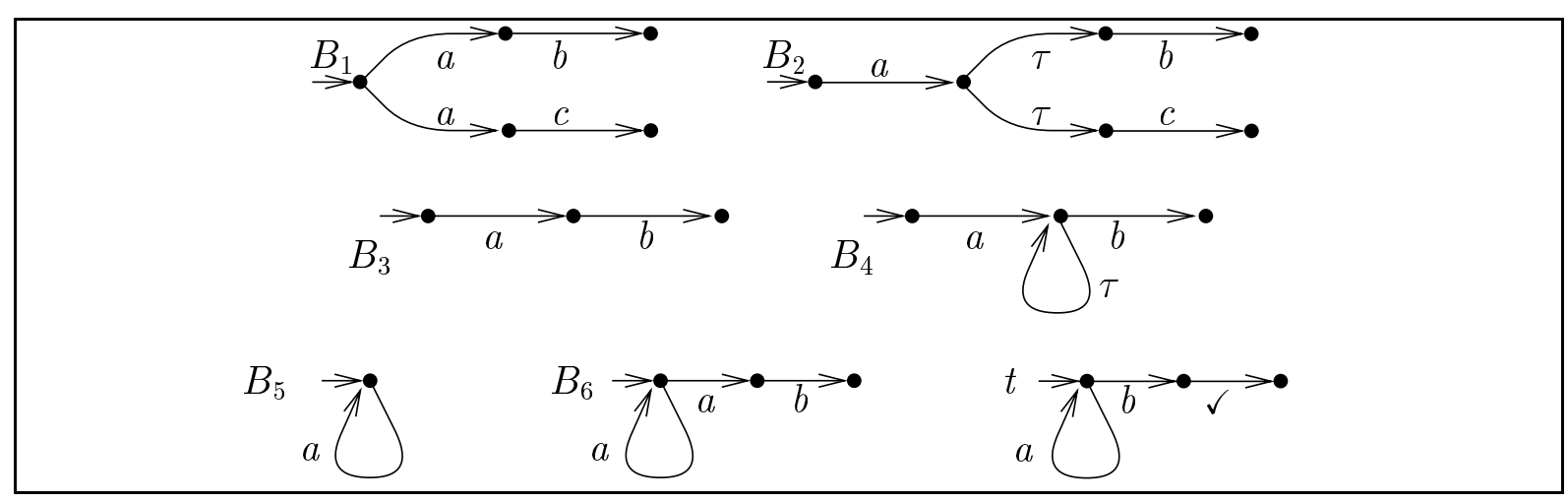

Figure 1 Transition system examples.

of equivalences are those that employ the notion of bisimulation (Milner (1989), Park (1981)), with observation congruence as one of its best-known members, and those that are induced by a formalised notion of testing (De Nicola and Hennessy (1984), Hennessy (1988), Brookes, Hoare and Roscoe (1984)). Bisimulations provide the finer equivalences that keep track of the branching structure of behaviours, and have a rather elegant proof theory. Testing equivalences are generally coarser and distinguish mainly on the basis of difference in deadlock behaviour, which is in practical cases often sufficient.

The higher resolution power of observation congruence is sometimes undesirable in practice. The transition systems $B_{1}$ and $B_{2}$ in Figure 1 are not observation congruent, but are testing equivalent. In practice, we would sometimes like to implement behaviour $B_{1}$ by $B_{2}$, see for example Groote (1988), resolving the choice between the two $a$-actions internally, and not in the interaction with the environment. A second advantage of testing equivalences is that they are generated by pre-orders that can be practically interpreted as implementation relations. They usually express that the implementation is some sort of deterministic reduction of the specified behaviour.

A feature of observation congruence is that it incorporates a particular notion of fairness. The behaviours $B_{3}$ and $B_{4}$ shown in Figure 1 are observation congruent. Observation congruence works on the principle that the $\tau$-loop of $B_{4}$ is executed an arbitrary but only finite number of times, in this case implying that eventually action $b$ will be enabled. Such identification of behaviour can be very useful in practice, for example, when proving properties of systems with lossy communication media. In such cases $\tau$-loops represent an unbounded but finite number of message losses. Interesting proofs of protocol correctness based on this principle can be found in Larsen and Milner (1987) and Brinksma (1995).

It is of practical interest to have a testing pre-order that combines the ability to shift and reduce nondeterminism with the capacity to model fairness. In particular, one would like to be compatible with observation congruence, since this would allow equalities demonstrated using the more elegant proof techniques of observation congruence to be preserved. At the same time, it is important for a relation to be pre-congruent with respect to all important combinators, i.e., the pre-order should be preserved when substituting related behaviours in a larger expression. This combination turns out to be non-trivial to achieve. Most of the standard testing-preorders are based on the interpretation of $\tau$-loops as divergences, which makes them quasi-observable as a chaotic or under-specified process, and therefore unfair. Although it is not that difficult to define a testing pre-order that shares the fairness property with observation congruence, viz. the fair must-testing of Brinksma (1988) (see 
also Section 3.3 of Vogler (1992)), pre-congruence with respect to hiding, which internalises visible actions and may thereby introduce new divergences, is then lost. For instance, in Figure 1, the behaviour $B_{5}$ has strictly fewer failures than $B_{6}$. After hiding $a$, the failure inclusion no longer holds: in a fair testing scenario, $B_{6}$ will always succeed in performing a $b$-action, whereas $B_{5}$ will refuse to do this. This shows that the removal of nondeterminism (taking away the $a-b$ branch in $B_{6}$ ) interferes with pre-congruence w.r.t. hiding.

In Brinksma, Rensink and Vogler (1995) we have proposed a solution to the longstanding problem of fair testing-preorders by introducing the notion of should-testing. This notion of testing, which was also independently developed in Natarajan and Cleaveland (1995), is based on a modification of the definition of a successful test: process $B$ passes test $t$ iff every finite execution of $B \| t$ (the process while tested) has passed through or can be extended to pass through a successful state of $t$. Using this definition, the tester process $t$ given in Figure 1 (with "success label" $\checkmark$ ) will distinguish between $B_{5}$ and $B_{6}$.

Based on this notion of testing Brinksma, Rensink and Vogler (1995) defines the preorder $\sqsubseteq_{\text {shd }}^{+}$, and proves that this is the coarsest pre-congruence for summation, abstraction and parallel composition contained in $\sqsubseteq_{\mathbf{f m s}}$, the fair must testing pre-order. In Brinksma, Rensink and Vogler (1996) we show that $\coprod_{\text {shd }}^{c}$, the intersection of $\coprod_{\text {shd }}^{+}$with trace equivalence, is the coarsest congruence for the fixpoint-operator contained in $\sqsubseteq_{\text {shd }}^{+}$. (Brinksma, Rensink and Vogler (1995) uses the notation $\sqsubseteq_{\text {shd }}^{c}$ to denote $\sqsubseteq_{\text {shd }}^{+}$.) In this paper we set out to show the application of $\sqsubseteq_{\text {shd }}^{c}$ to a number of examples that show the practical advantages over standard observation equivalence and (fair) must-testing. The structure is as follows: Section 2 contains the main definitions and notations, Section 3 discusses a number of proof principles for $\sqsubseteq_{\text {shd }}^{c}$ that we need. Section 4 contains the applications, which include a scheduling problem, a version of the AB-protocol, and fair communication channels. Section 5 summarises the work and presents our conclusions.

\section{DEFINITIONS}

\subsection{Language}

We assume countable sets $\mathbf{A}$ of actions, ranged over by $a, b, \ldots$, and $\mathbf{X}$ of process names, ranged over by $X, Y, Z$. There is also a special invisible action $\tau \notin \mathbf{A}$; we denote $\mathbf{A}_{\tau}=$ $\mathbf{A} \cup\{\tau\}$, ranged over by $\alpha, \beta, \ldots$. We consider a language $\mathbf{L}$ with the following grammar:

\section{$B::=\alpha ; B \mid \sum$ setof $B\left|B \|_{A} B\right| B[\phi]|B / A| X$.}

Hence, the language features a family of action prefix operators ( $\alpha \in \mathbf{A}_{\tau}$ arbitrary), a CCS-like infinitary summation operator $\sum$, a CSP-like parallel composition operator indexed by the set of synchronisation actions ( $A \subseteq \mathbf{A}$ arbitrary), a renaming operator indexed by a function $(\phi: \mathbf{A} \rightarrow \mathbf{A}$ arbitrary, extended with $\tau \mapsto \tau)$, a hiding operator indexed by the set of actions to be abstracted away from ( $A \subseteq \mathbf{A}$ arbitrary) and process invocation. Furthermore, we use abbreviated forms of summation and synchronisation:

$$
\text { stop }=\sum \varnothing \quad B_{1}+B_{2}=\sum\left\{B_{1}, B_{2}\right\} \quad B+\sum \mathbf{B}=\sum(\mathbf{B} \cup\{B\}) \quad B_{1}\left\|B_{2}=B_{1}\right\|_{\mathbf{A}} B_{2}
$$


Table 1 Structural operational semantics of $\mathbf{L}$

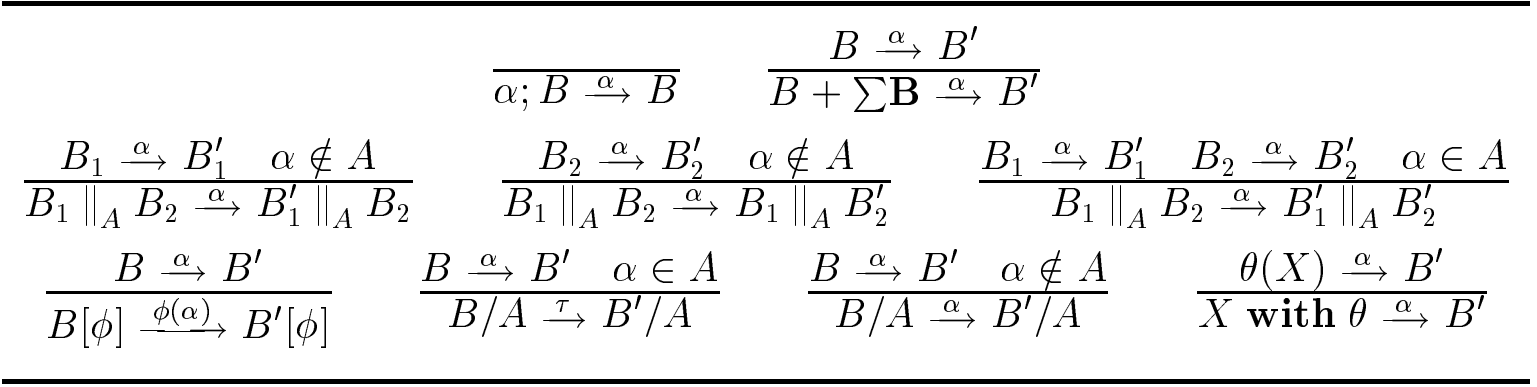

We take the operators of $\mathbf{L}$ to be sufficiently familiar to make an extensive discussion superfluous. Terms of $\mathbf{L}$ are interpreted in the context of a process environment $\theta: \mathbf{X} \rightarrow \mathbf{L}$, which is such that for every $X, Y \in \mathbf{X}, X$ is guarded in $\theta(Y)$. We often write $X={ }_{\theta} B$ to indicate $\theta(X)=B$. $\theta$ defines the meaning of the process names, in the sense that whenever a process name is encountered, its $\theta$-image is taken. We sometimes explicitly write $B$ with $\theta$ to indicate that $B$ is to be interpreted in the environment $\theta$; and we write

$\theta_{X}^{B}: Y \mapsto \begin{cases}B & \text { if } Y=X \\ \theta(Y) & \text { otherwise. }\end{cases}$

The operational semantics of $\mathbf{L}$ is entirely standard; its definition, through structural operational rules, is recalled in Table 1. Each $B \in \mathbf{L}$ therefore gives rise to an $\mathbf{A}_{\tau}$-labelled transition system $\langle\mathbf{L}, \rightarrow, B\rangle$, where $\rightarrow \subseteq \mathbf{L} \times \mathbf{A}_{\tau} \times \mathbf{L}$ is the the transition relation induced by the operational rules. In general, if $\langle S, \rightarrow, q\rangle$ is an $\mathbf{A}_{\tau}$-labelled transition system then we define weak transitions in the usual fashion: for all $s, s^{\prime} \in S$,

$s \stackrel{a_{1} \cdots a_{n}}{\longrightarrow} s^{\prime}: \Leftrightarrow s \stackrel{\tau}{\longrightarrow} \stackrel{a_{1}}{\longrightarrow} \stackrel{\tau}{\longrightarrow} \cdots \stackrel{\tau}{\longrightarrow} \stackrel{a_{n}}{\longrightarrow} \stackrel{\tau}{\longrightarrow}^{*} s^{\prime}$

\subsection{Testing and observation}

In this paper we study testing preorders based on the operational semantics. This entails a setup wherein systems are investigated by synchronising them with tests, which are terms of an extended language $\mathbf{L}_{\checkmark}$ that contains the special success action $\checkmark \notin \mathbf{A}_{\tau}$. Tests will be ranged over by $t$; their operational semantics is again determined by Table 1 , by interpreting $\alpha$ as ranging over $\mathbf{A}_{\tau} \cup\{\checkmark\}$. We denote $\mathbf{A}_{\checkmark}=\mathbf{A} \cup\{\checkmark\}$.

Applying the test $t$ to the process $B$ results in the term $B \| t$. Whether or not such a test application is deemed successful depends on the choice of test modality; for the purpose of this paper we distinguish may-, fair must- and should-testing. ${ }^{2}$

$$
\begin{aligned}
B \text { may } t & : \Leftrightarrow \quad \exists w \in \mathbf{A}^{*} .(B \| t) \stackrel{w \checkmark}{=} \\
B \text { fms } t & : \Leftrightarrow \quad \forall w \in \mathbf{A}^{*}, \forall B^{\prime} .(B \| t) \stackrel{w}{\Longrightarrow} B^{\prime} \text { implies } \exists \alpha \in \mathbf{A}_{\checkmark} . B^{\prime} \stackrel{\alpha}{\Longrightarrow} \\
B \text { shd } t & : \Leftrightarrow \quad \forall w \in \mathbf{A}^{*}, \forall B^{\prime} .(B \| t) \stackrel{w}{\Longrightarrow} B^{\prime} \text { implies } \exists v \in \mathbf{A}^{*} . B^{\prime} \stackrel{v \checkmark}{\Longrightarrow} .
\end{aligned}
$$

\footnotetext{
${ }^{2}$ Because we are interested in fairness, we have omitted from this list divergence-sensitive testing modalities, most prominently among which is the well-known must-testing of De Nicola and Hennessy (1984).
} 
In addition, we use a stability test: $B$ stb $: \Leftrightarrow B \stackrel{\tau}{\longrightarrow}$. The corresponding testing preorders express that the left-hand system (the proposed implementation) passes all the tests that the right-hand system (the given specification) passes, in the sense of the chosen testing modality.

$$
\begin{aligned}
I \sqsubseteq \bmod S & : \Leftrightarrow \quad \forall t \in \mathbf{L}_{\checkmark} . S \bmod t \Longrightarrow I \bmod t \quad(\bmod =\operatorname{may}, \mathbf{f m s}, \mathbf{s h d}) \\
I \sqsubseteq \mathbf{s t b} & : \Leftrightarrow \quad S \mathbf{s t b} \Longrightarrow I \mathbf{s t b} .
\end{aligned}
$$

Each of these relations can be characterised more directly in terms of the operational behaviour of the systems. For this purpose, we define the language $L$, failures $F$ and tree refusals $R$ of a state $s \in S$ of a transition system $T=\langle S, \rightarrow, q\rangle$ :

$$
\begin{aligned}
& L_{T}(s):=\left\{w \in \mathbf{A}^{*} \mid \exists s^{\prime} . s \stackrel{w}{\Longrightarrow} s^{\prime}\right\} \\
& F_{T}(s):=\left\{(w, A) \in L_{T}(s) \times \mathcal{P}(\mathbf{A}) \mid \exists s^{\prime} . s \stackrel{w}{\Longrightarrow} s^{\prime} \wedge L_{T}\left(s^{\prime}\right) \cap A=\varnothing\right\} \\
& R_{T}(s):=\left\{X \subseteq L_{T}(s) \mid \exists w \in \downarrow X . \exists s^{\prime} . s \stackrel{w}{\Longrightarrow} s^{\prime} \wedge L_{T}\left(s^{\prime}\right) \cap w^{-1} X=\varnothing\right\} .
\end{aligned}
$$

We also write $L(T)$ for $L_{T}(q)$ etc. The definition of $R$ uses auxiliary notations for the prefix closure of a set of strings $X$, resp. the remainder of $X$ after some string $w$ :

$$
\begin{aligned}
\downarrow X & :=\left\{w \in \mathbf{A}^{*} \mid \exists v \in \mathbf{A}^{*} . w v \in X\right\} \\
w^{-1} X & :=\left\{v \in \mathbf{A}^{*} \mid w v \in X\right\} .
\end{aligned}
$$

Hence, the tree refusals in $R_{T}$ are a generalisation of the failures in $F_{T}$, in that the initial trace $w$ is not fixed but an arbitrary member of $\downarrow X$, and not single actions but entire traces are refused $\left(L_{T}\left(s^{\prime}\right) \cap w^{-1} X=\varnothing\right.$ rather than $\left.L_{T}\left(s^{\prime}\right) \cap A=\varnothing\right)$.

Proposition 1 The following characterisations hold:

$$
\begin{aligned}
I \sqsubseteq \text { may } & \Longleftrightarrow \quad L(I) \supseteq L(S) \\
I \sqsubseteq_{\text {fms }} S & \Longleftrightarrow F(I) \subseteq F(S) \\
I \sqsubseteq_{\text {shd }} S & \Longleftrightarrow L(I) \subseteq L(S) \wedge R(I) \subseteq R(S) .
\end{aligned}
$$

For the proofs see, respectively, De Nicola and Hennessy (1984), Brinksma (1988), Vogler (1992) and Brinksma, Rensink and Vogler (1995). In addition to the above testing preorders, we make use of the well-known notion of observation congruence (Milner (1989)). Let $\hat{\imath}: \mathbf{A}_{\tau} \rightarrow \mathbf{A}^{*}$ be a mapping defined by $\hat{a}=a$ for all $a \in \mathbf{A}$ and $\hat{\tau}=\varepsilon$.

Definition 2 (observation congruence) Let $\langle S, \rightarrow, q\rangle$ be a transition system.

- Observation equivalence $\approx \subseteq S \times S$ is the largest symmetrical relation such that for all $s_{1} \approx s_{2}$ and $s_{1} \stackrel{\alpha}{\longrightarrow} s_{1}^{\prime}$ there is a $s_{2}^{\prime} \approx s_{1}^{\prime}$ such that $s_{2} \stackrel{\hat{\alpha}}{\Longrightarrow} s_{2}^{\prime}$.

- Observation congruence $\simeq_{\mathbf{o b s}} \subseteq S \times S$ is the largest symmetrical relation such that for all $s_{1} \simeq$ obs $s_{2}$ and $s_{1} \stackrel{\alpha}{\longrightarrow} s_{1}^{\prime}$ there is a $s_{2}^{\prime} \approx s_{1}^{\prime}$ such that $s_{2} \stackrel{\alpha}{\Longrightarrow} s_{2}^{\prime}$.

The proof technique of observation congruence is much different from that of testing; as it happens, it is appreciably less complex. The relation between these various notions of 
correctness in terms of their mutual strength are recalled in the following proposition; the proofs follow from Proposition 1 or can be found in Brinksma, Rensink and Vogler (1995).

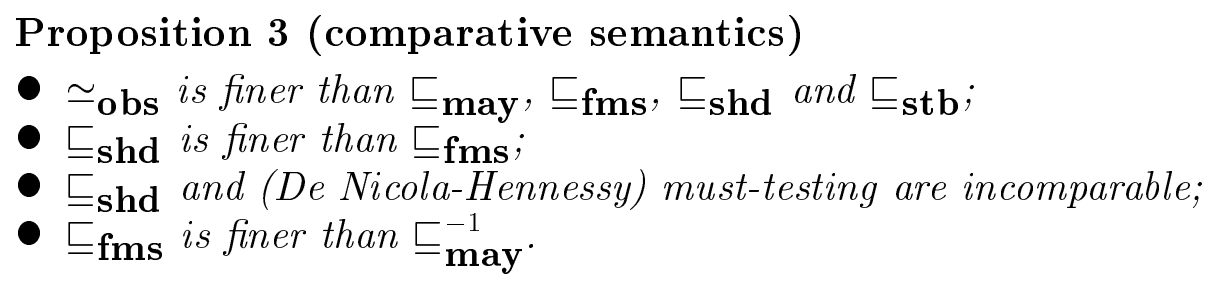

\subsection{Congruence}

The concept of (pre-)congruence with respect to a language is usually defined in terms of contexts, which are terms of $\mathbf{L}$ possibly containing a "hole" denoted [], interpreted in a process environment $\theta$ which may also contain such holes. If $C$ [ ] denotes such a context, then $C[B]$ denotes the term obtained by substituting $B$ for all the occurrences of [ ] in $C$ and $\theta$. Furthermore, we define recursive pre-congruence, which means that related terms may also be substituted in process environments.

Definition 4 Let $\sqsubseteq$ be a preorder over $\mathbf{L}$.

- $\sqsubseteq$ is pre-congruent for [a fragment of] $\mathbf{L}$ if for all contexts expressed in [that fragment of] $\mathbf{L}, I \sqsubseteq S$ implies $C[I] \sqsubseteq C[S]$.

- $\sqsubseteq$ is recursively pre-congruent if it is pre-congruent and $I$ with $\theta_{X}^{B} \sqsubseteq S$ with $\theta_{X}^{B}$ for all $B$ implies $X$ with $\theta_{X}^{I} \sqsubseteq X$ with $\theta_{X}^{S}$.

As an example of recursive pre-congruence, consider that the axiom $B+B=B$ for a recursively congruent relation implies $X$ with $\theta=X$ with $\theta_{X}^{\theta(X)+\theta(X)}$. Other examples can be found in Section 4.3. A relation is mathematically tractable only if it is a recursive pre-congruence. The following congruence properties are known or easily shown to hold:

\section{Proposition 5}

- De Nicola and Hennessy (1984), Milner (1989): $\sqsubseteq_{\mathbf{m a y}}, \simeq_{\mathbf{o b s}}$ and the combination of $\sqsubseteq_{\mathbf{m a y}}$ and $\sqsubseteq_{\mathbf{s t b}}$ are recursively pre-congruent for $\mathbf{L}$;

- Brinksma (1988), Vogler (1992): The combination of $\sqsubseteq_{\mathbf{f m s}}$ and $\sqsubseteq_{\mathbf{s t b}}$ is pre-congruent for the fragment of $\mathbf{L}$ not containing hiding;

- Brinksma, Rensink and Vogler (1996): The combination of $\sqsubseteq_{\mathbf{m a y}}$, $\sqsubseteq_{\mathbf{s h d}}$ and $\sqsubseteq_{\mathbf{s t b}}$ is recursively pre-congruent for $\mathbf{L}$; in fact, it is the coarsest recursive pre-congruence within $\sqsubseteq_{\mathbf{f m s}}$.

In the remainder of this paper, we concentrate on the last relation addressed in the above proposition; that is, we will use $\sqsubseteq_{\text {shd }}^{c}$ defined by

$I \sqsubseteq_{\text {shd }}^{c} S: \Leftrightarrow I \sqsubseteq_{\text {may }} S \wedge I \sqsubseteq_{\text {shd }} S \wedge I \sqsubseteq_{\text {stb }} S$.

The notation $\sqsubseteq_{\text {shd }}^{c}$ is intended to convey that the relation is the coarsest congruence contained in $\sqsubseteq_{\mathbf{s h d}}$, as Proposition 5 implies. We also write $\simeq_{\text {shd }}^{c}$ for the equivalence 
generated by $\sqsubseteq_{\text {shd }}^{c}$. From Proposition 1 it follows that the following denotational characterisation holds — which allows to apply $\sqsubseteq_{\text {shd }}^{c}$ also to transition systems:

$I \sqsubseteq_{\text {shd }}^{c} S \Longleftrightarrow L(I)=L(S) \wedge R(I) \subseteq R(S) \wedge(I \stackrel{\tau}{\longrightarrow} \Longrightarrow S \stackrel{\tau}{\longrightarrow})$.

\section{PROOF PRINCIPLES}

We discuss a number of general methods to prove that the $\sqsubseteq_{\mathbf{s h d}}^{c}$-preorder holds between a given pair of systems, avoiding the direct use of the (costly) denotational characterisation as much as possible. In the next section, we use these methods in a number of examples of system specifications and their implementations on the basis of fair testing.

\subsection{The bisimulation inheritance}

Since observation congruence is stronger than all our testing pre-orders (see Proposition 3), all known methods to prove $\simeq_{\mathbf{o b s}}$ are valid for proving $\simeq_{\text {shd }}^{c}$. This is an advantage because, as mentioned before, the proof techniques for observation congruence are relatively simple; hence, if it holds, it is cheaper to show $\simeq$ obs than to try to show $\simeq_{\text {shd }}^{c}$ directly. Of course, it may be that observation congruence fails to hold between two given $\sqsubseteq_{\text {shd }}^{c}$ related systems, in which case the inherited proof techniques obviously cannot work. If this is so, then one can still try methods more directly tuned to the testing notion one is actually interested in. (This point is made very forcefully by Valmari (1995) in the context of transition system reduction.)

Of the proof techniques available for observation congruence we mention two: constructing a bisimulation relation and applying the equational theory. The details of bisimulation relations are omitted here; see Milner (1989) for an exposition. With respect to the equational theory, we recall axioms in Table 2, adapted from Milner to our setting. Axioms (3)-(5) explain, respectively, synchronisation, renaming and hiding in terms of action prefix and choice. In fact, using (2)-(5) one may rewrite every finite term into a term of the form $\sum_{i \in I} \alpha_{i} ; B_{i}$ where the $B_{i}$ are again of this form. Axiom (6), on the other hand, states that we may always replace process names by their definitions.

The above axioms in fact hold up to strong bisimilarity, which is finer yet than observation congruence. Observation congruence additionally satisfies the so-called $\tau$-laws (7)-(9). Finally, observation congruence also satisfies the so-called Definition Fair Abstraction Rule DFAR, which is one way to express the fact that the relation is fair: ${ }^{3}$

$\frac{X_{i}={ }_{\theta} a_{i \oplus 1} ; X_{i \oplus 1}+B_{i} \text { for } 0 \leq i<n}{X_{i} / A \text { with } \theta=\tau ; \sum_{0 \leq i<n}\left(B_{i} / A\right) \text { with } \theta} A=\left\{a_{i} \mid 0 \leq i<n\right\}$

${ }^{3}$ DFAR is subtly different from the original Koomen's Fair Abstraction Rule (KFAR) in Baeten and Weijland (1990): where the premise of DFAR concerns the definition of the $X_{i}$, KFAR has semantic equality in the premisse. In fact, KFAR is implied by DFAR plus the so-called recursive specification principle (RSP), which states that sets of guarded equations have a unique solution. Since neither RSP nor KFAR holds for $\Xi_{\text {shd }}^{c}$, we have to restrict to the weaker DFAR. See Brinksma, Rensink and Vogler (1995) for a more extensive discussion. 
Table 2 Axioms of strong bisimilarity, with $B=\sum_{i \in I} \beta_{i} ; B_{i}$ and $C=\sum_{k \in K} \gamma_{k} ; C_{k}$ :

$$
\begin{aligned}
& B=\sum\{B\} \\
& B \|_{A} C=\sum_{\beta_{i} \notin A} \beta_{i} ;\left(B_{i} \|_{A} C\right)+\sum_{\gamma_{k} \notin A} \gamma_{k} ;\left(B \|_{A} C_{k}\right)+\sum_{\beta_{i}=\gamma_{k} \in A} \beta_{i} ;\left(B_{i} \|_{A} C_{k}\right) \\
& B[\phi]=\sum_{i \in I} \phi\left(\beta_{i}\right) ;\left(B_{i}[\phi]\right) \\
& B / A=\sum_{\beta_{i} \in A} \tau ;\left(B_{i} / A\right)+\sum_{\beta_{i} \notin A} \beta_{i} ; B_{i} / A \\
& X=\theta(X)
\end{aligned}
$$

\begin{tabular}{|c|c|}
\hline$\alpha ; \tau ; B=\alpha ; B$ & $(7)$ \\
\hline$B+\tau ; B=\tau ; B$ & $(8)$ \\
\hline$\alpha ;(B+\tau ; C)+\alpha ; C=\alpha ;(B+\tau ; C)$ & $(9)$ \\
\hline \multicolumn{2}{|c|}{$\tau$-laws of should-testing: } \\
\hline$\alpha ; B+\alpha ; C=\alpha ;(\tau ; B+\tau ; C)$ & $(10)$ \\
\hline$\tau ;(B+C) \sqsubseteq B+\tau ; C$ & $(11)$ \\
\hline$\alpha ; B+\tau ;(\alpha ; C+D)=\tau ;(\alpha ; B+\alpha ; C+D)$ & $(12)$ \\
\hline$B \sqsubseteq \tau ; B$ & $(13)$ \\
\hline if $B \sqsubseteq C$ then $C \sqsubseteq \tau ; B+\tau ; C$ & $(14)$ \\
\hline
\end{tabular}

$\tau$-laws of observation congruence:

(Here $\oplus$ denotes addition modulo $n$.) Note that the equality sign in the premise does not refer to the semantic relation to which the rule is applied (observation congruence, in this case) but rather to the definition of the process environment $\theta$. Therefore the rule also applies to any relation weaker than observation congruence, such as $\sqsubseteq_{\text {shd }}^{c}$.

Among other things, DFAR in combination with Axiom (7) can be used to show that $\tau$-loops at non-initial states can be ignored: if $X={ }_{\theta} a ; X+B$ for some $a$ not occurring in $B$ then $\alpha ;(X / a) \simeq_{\mathbf{o b s}} \alpha ; \tau ;(B / a) \simeq_{\mathbf{o b s}} \alpha ; B$.

\subsection{The testing theory}

We do not have a complete equational theory of $\sqsubseteq_{\text {shd }}^{c}$. However, there are a number of axioms that this relation satisfies beyond those of observation congruence. In fact, although $\sqsubseteq_{\text {shd }}^{c}$ is incomparable to the standard must-testing of De Nicola and Hennessy (1984) (see Proposition 3), most of their axioms dealing with nondeterminism do hold in our setting as well: see Table 2. Rule (14) comes in the place of $B \sqsubseteq \tau ; B+\tau$; $C$, which is an important axiom of must-testing that is not satisfied by $\sqsubseteq_{\text {shd }}^{c}$ (it contradicts the language equality implicit in $\sqsubseteq_{\text {shd }}^{c}$; see (1)). 


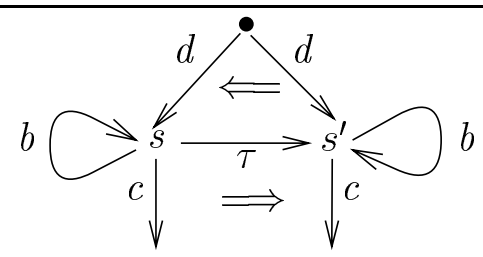

Figure 2 preconditions for contraction

Theorem $6 \sqsubseteq_{\text {shd }}^{c}$ satisfies Axioms (10)-(13) and Rule (14).

Note that these axioms (together with the idempotence of choice, which is derivable in our setting) imply the $\tau$-laws of observation congruence.

\subsection{Denotational arguments}

As remarked before, the equational theory presented above is not complete. This means that occasionally one may be forced to show that two systems are $\Xi_{\text {shd }}^{c}$-related by directly accessing the denotational characterisation. As an example of a property proved in this way, we formulate a contraction lemma stating that under certain circumstances, two states of a transition system can be identified.

Lemma 7 (contraction lemma) Let $T=\langle S, \rightarrow, q\rangle$ be a transition system with states $s \neq q \neq s^{\prime}$ that satisfy the following conditions (see Figure 2):

a) $s \stackrel{\alpha}{\longrightarrow} s^{\prime}$ iff $\alpha=\tau$;

b) $s \stackrel{a}{\rightarrow} s$ iff $s^{\prime} \stackrel{a}{\rightarrow} s^{\prime}$;

c) if $s \stackrel{\alpha}{\longrightarrow} t$ for some $t \in S \backslash\left\{s, s^{\prime}\right\}$, then $s^{\prime} \stackrel{\alpha}{\longrightarrow} t^{\prime}$ for some $t^{\prime} \in S \backslash\left\{s^{\prime}\right\}$;

d) if $t \stackrel{\alpha}{\longrightarrow} s^{\prime}$ for some $t \in S \backslash\left\{s, s^{\prime}\right\}$, then $t \stackrel{\alpha}{\longrightarrow} s$, too.

Let $U=\left\langle S^{\prime}, \rightarrow, q\right\rangle$ be obtained from $T$ by contracting $s$ and $s^{\prime}$ to s, i.e., by putting $S^{\prime}=S \backslash\left\{s^{\prime}\right\}$ and replacing $s^{\prime}$ in the arcs by s. Then $T \simeq_{\text {shd }}^{c} U$.

Proof. Using the denotational characterisation (Proposition 1). Omitted; see Brinksma, Rensink and Vogler (1996).

An algebraic counterpart of the above lemma is difficult to give. Another denotational argument is presented in the following proposition. We call a transition system deterministic if for all reachable states $s, s \stackrel{\alpha}{\longrightarrow} s_{1}$ and $s \stackrel{\alpha}{\longrightarrow} s_{2}$ implies $\alpha \neq \tau$ and $s_{1}=s_{2}$.)

Proposition 8 If $S$ is deterministic and $I \sqsubseteq_{\text {shd }}^{c} S$ then $I \simeq$ obs $S$.

Proof. The determinism of $S$ implies $R(S)=\varnothing$, hence $R(I)=\varnothing$; the absence of $\tau$-moves implies stability of $S$ and hence of $I$. From this one can prove that $I^{\prime} \approx S^{\prime}$ whenever $I \stackrel{w}{\Longrightarrow} I^{\prime}$ and $S \stackrel{w}{\Longrightarrow} S^{\prime}$; due to the stability of $I$ and $S$ it then follows that $I \simeq$ obs $S$.

As a final "denotational" proof technique, we mention the following result, the proof of which is contained in Brinksma, Rensink and Vogler (1996):

Theorem $9 \sqsubseteq$ shd is decidable for finite-state systems. 

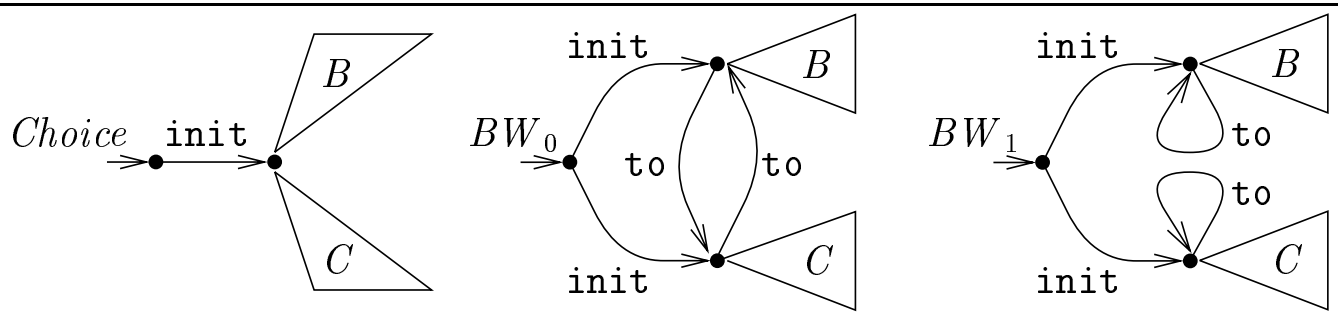

Figure 3 Correct and incorrect versions of busy waiting (to abbreviates timeout).

\subsection{Compositionality}

In conjunction with the methods presented above, it is very important to realise that, due to the fact that our notion of implementation is pre-congruent, proofs of correctness can be done in a compositional manner. That is, it is not necessary to consider complete systems; rather, one may take a single module and replace that by a "better" one (more fault-tolerant, more deterministic, etc.); the entire system will thereby be improved, and the result of this replacement is a formal implementation of the original system.

\section{EXAMPLES}

The purpose of the following is to demonstrate the advantages of shd-testing compared to observation congruence, fms- and divergence-sensitive must-testing.

\subsection{External choice as busy-waiting}

Our first, simple example concerns the implementation of external choice as busy waiting; see Figure 3. The process Choice $={ }_{\theta}$ init; $(B+C)$ states that, after initialisation, either $B$ or $C$ can be chosen. A busy-waiting implementation oscillates between $B$ and $C$ :

$$
\begin{array}{rll}
B W_{0} & ={ }_{\theta} & \text { init; }_{\text {Wait }_{B}}+\text { init; }_{\text {Wait }_{C}} \\
\text { Wait }_{B} & ={ }_{\theta} & \text { B }+ \text { timeout; }_{\text {Wait }} \\
\text { Wait }_{C} & ={ }_{\theta} & C+\text { timeout; }_{\text {Wait }} \\
B
\end{array}
$$

where timeout is assumed not to occur in $B$ or $C$ (hence $B /$ timeout $\simeq$ obs $B$ and $C /$ timeout $\simeq$ obs $C$ ). From DFAR (15) and Axiom (7), it follows that Choice $\simeq_{\text {obs }}$ $B W_{0} /$ timeout; hence this also holds for $\simeq_{\mathbf{s h d}}^{c}$ and $\simeq_{\mathbf{f m s}}$ (Proposition 3 ). The systems are not equivalent under De Nicola-Hennessy must-testing, since this is sensitive to the divergence in $B W_{0} /$ timeout. To show the advantage of $\simeq_{\text {shd }}^{c}$ over $\simeq_{\mathbf{f m s}}$, consider

$$
\begin{aligned}
& B W_{1}=_{\theta} \quad \text { init; } \text { Wait }_{B}^{\prime}+\text { init; } \text { Wait }_{C}^{\prime} \\
& \text { Wait }_{B}^{\prime}={ }_{\theta} \quad B+\text { timeout; } \text { Wait }_{B}^{\prime} \\
& \text { Wait }_{C}^{\prime}={ }_{\theta} \quad C+\text { timeout; Wait }{ }_{C}^{\prime} \text {. }
\end{aligned}
$$

$B W_{1}$ fails to change between options, so $B W_{1} /$ timeout is certainly not a correct implementation of Choice. Accordingly, $B W_{0} \not_{\text {shd }}^{c} B W_{1}$; but fair must-testing suggests that 

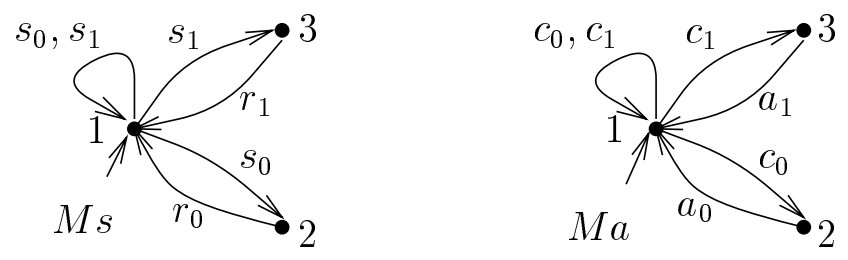

Figure 4 Two lossy channels.

$B W_{0}$ and $B W_{1}$ can be used interchangeably, since $B W_{0} \simeq_{\mathbf{f m s}} B W_{1}$ (any failure of $B W_{0}$ can be found in $B W_{1}$ and vice versa, by taking the appropriate initial init-branch). This, therefore, is an example where the lack of pre-congruence w.r.t. hiding makes fair must-testing unsuitable.

\subsection{The alternating bit protocol}

As a more extensive example we will use $\simeq_{\text {shd }}^{c}$ to show the correctness of a version of the alternating-bit protocol. The desired behaviour is that of a one-place buffer:

$B u f={ }_{\theta}$ snd; rcv; Buf,

where we abstract from the content of the message sent. The implementation is built from the sender $S n d_{0}$ and the receiver $R c v_{0}$, which are connected by two lossy channels $M s$ and $M a$ for transmitting messages and acknowledgements. An additional bit is appended to the messages and acknowledgements, so $M s$ participates in sending with $s_{i}$ on the one side and in receiving with $r_{i}$ on the other, while $M a$ participates in confirming with $c_{i}$ and in acknowledging with $a_{i}(i=0,1)$. The channels are given by

$$
\begin{aligned}
& M s={ }_{\theta} \quad s_{0} ; r_{0} ; M s+s_{0} ; M s+s_{1} ; r_{1} ; M s+s_{1} ; M s \\
& M a={ }_{\theta} \quad c_{0} ; a_{0} ; M a+c_{0} ; M a+c_{1} ; a_{1} ; M a+c_{1} ; M a
\end{aligned}
$$

(see Figure 4). Since $M s$ can repeatedly lose the message, which at best leads to a possibly infinite repetition of this message, it is clear that an implementation on this basis will be able to diverge instead of delivering the message. Hence, such an implementation cannot be correct with respect to a divergence-sensitive relation like De Nicola-Hennessy musttesting. In contrast, $\sqsubseteq_{\mathbf{f m s}}, \sqsubseteq_{\mathbf{s h d}}^{c}$ and $\simeq_{\mathbf{o b s}}$ can ignore divergence due to their built-in fairness assumption; and indeed, our implementation will be correct for each of these.

In this implementation, the sender $S n d_{0}$ gets a message with snd, appends the bit 0 , forwards it with $s_{0}$ to the receiver and waits in state $A c k_{0}$ for an acknowledgement. In this state, the sender may accept an acknowledgement $a_{1}$ with the wrong bit, but will ignore it; the sender may repeat the message; upon getting the correct acknowledgement $a_{0}$, it will repeat its behaviour using bit 1 . The receiver works analogously, starting in state $R c v_{0}$ where it waits for the first reception. Sender and receiver are defined by:

$$
\begin{array}{ll}
\operatorname{Snd}_{i}={ }_{\theta} & \text { snd; } s_{i} ; A c k_{i} \\
\text { Ack }_{i}={ }_{\theta} & a_{i} ; \operatorname{Snd}_{1-i}+a_{1-i} ; A c k_{i}+s_{i} ; A c k_{i} \\
\text { Rcv }_{i}={ }_{\theta} & r_{i} ; \mathrm{rcv} ; c_{i} ; \text { Cnf }_{i}
\end{array}
$$




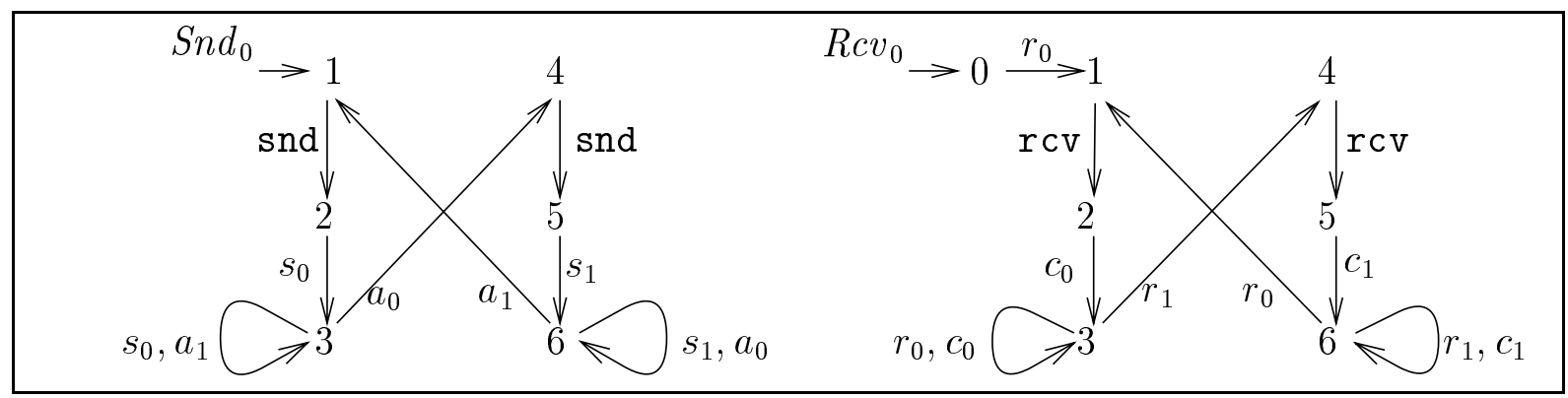

Figure 5 Sender and receiver.

$C n f_{i}={ }_{\theta} \quad \operatorname{Rcv}_{1-i}+r_{i} ; \operatorname{Cnf}_{i}+c_{i} ; \operatorname{Cnf}_{i}$

(see Figure 5). In the implementation, the four components are composed using suitable synchronisation sets, and all actions except snd and $\mathrm{rcv}$ are hidden, i.e.

$A B P={ }_{\theta}\left(\left(S n d_{0} \|_{s_{0}, s_{1}} M s\right) / s_{0}, s_{1} \|_{r_{0}, r_{1}, a_{0}, a_{1}}\left(R c v_{0} \|_{c_{0}, c_{1}} M a\right) / c_{0}, c_{1}\right) / r_{0}, r_{1}, a_{0}, a_{1}$.

We will show $B u f \simeq_{\text {shd }}^{c} A B P$ in a compositional fashion, viz. by reducing subsystems of $A B P$ while building up its transition system, which in the end will be checked against the specification. First, we compose $S n d_{0}$ with $M s$ synchronising over $s_{0}, s_{1}$. The resulting transition system is shown in Figure 6, where a state $i j$ corresponds to $S n d_{0}$ being in state $i$ according to Figure 5 and $M s$ being in state $j$ according to Figure $4 . s_{0}$ and $s_{1}$ are subsequently hidden.

For the reduction of the resulting system we can use Lemma 7 of the previous section, by applying it to $s=31$ and $s^{\prime}=32$ on the one hand and to $s=61$ and $s^{\prime}=63$ on the other. Afterwards, we can omit the $\tau$-loops at the contracted states according to DFAR (15). Consequently, there is only one arc leaving 21 and this is a $\tau$-arc; according to (7), we can contract this arc and similarly for 51 . The resulting system is shown in Figure 7 with a new enumeration of the states.

Note that this transition system is not observation congruent to the one in Figure 6 (with $s_{0}$ and $s_{1}$ hidden). The latter can perform snd to reach 32 from which $a_{0}$ can always be followed by $r_{0}$. The former necessarily reaches 2 when performing snd, and from there

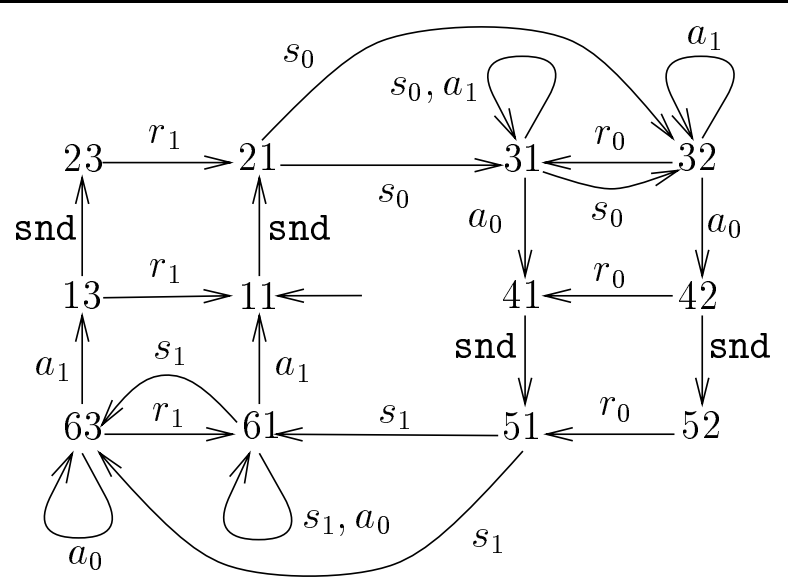

Figure 6 Sender composed with message channel. 


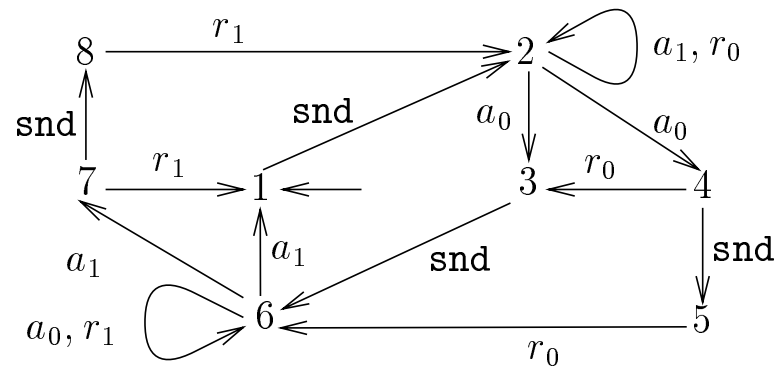

Figure 7 Sender and message channel after contraction.

$a_{0}$ can be performed such that $r_{0}$ is impossible. Hence, the reduction discussed above is not valid up to $\simeq_{\mathbf{o b s}}$; indeed, Lemma 7 fails for $\simeq$ obs .

Similarly as above, we construct the transition system of $R c v_{0} \|_{\left\{c_{0}, c_{1}\right\}} M a$ and reduce it after hiding $c_{0}$ and $c_{1}$ (Figure 8). As a final step, we compose the systems of Figures 7 and 8 to get the transition system of $\left(S n d_{0} \|_{s_{0}, s_{1}} M s\right) / s_{0}, s_{1} \|_{r_{0}, r_{1}, a_{0}, a_{1}}\left(R c v_{0} \|_{c_{0}, c_{1}} M a\right) / c_{0}, c_{1}$ reduced up to $\simeq_{\text {shd }}^{c}$; see Figure 9 .

Now one can show observation congruence of Buf with the system in Figure 9 after hiding $r_{0}, r_{1}, a_{0}, a_{1}$. Informally, from the start state 10 we reach 22 by performing snd and rcv; from 22 , the system moves to 62 with snd, from 62 to 66 with rcv, and so on. Formally:

$s \approx B u f \quad$ for $s=10,16,22,28,32,42,65,66,76$

$s \approx \mathrm{rcv} ;$ Buf for $s=20,21,26,27,52,62,63,64,86$

and hence $A B P \simeq_{\text {shd }}^{c} 10 \simeq$ obs $B u f$, implying $A B P \simeq_{\text {shd }}^{c} B u f$. Because $B u f$ is clearly stable and deterministic, from Proposition 8 it follows that $A B P \simeq$ obs $B u f$.

\subsection{Alternative channels}

To further stress the advantages of $\simeq_{\text {shd }}^{c}$, we will discuss the effect of changing the behaviour of the channel $M s$. For this purpose, we define a number of contexts.

$$
\begin{aligned}
& C_{1}[]=s_{0} ;\left(\tau ; r_{0} ;[]+\tau ;[]\right)+s_{1} ;\left(\tau ; r_{1} ;[]+\tau ;[]\right) \\
& C_{2}[]=s_{0} ;\left(r_{0} ;[]+[]\right)+s_{1} ;\left(r_{1} ;[]+[]\right) \\
& C_{3}[]=s_{0} ;\left(r_{0} ;[]+\tau ;[]\right)+s_{1} ;\left(r_{1} ;[]+\tau ;[]\right)
\end{aligned}
$$

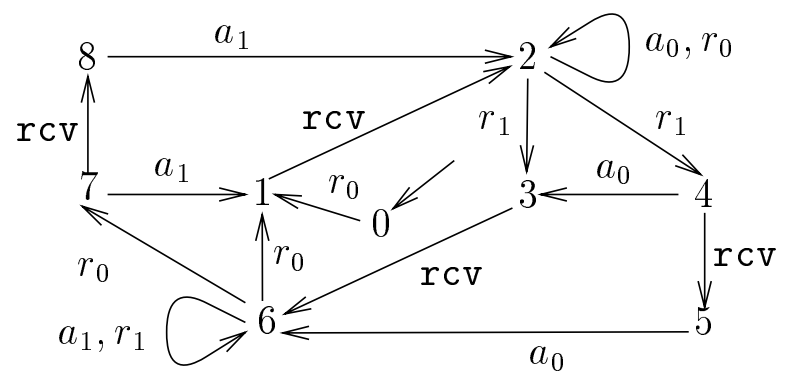

Figure 8 Receiver and acknowledgement channel after contraction. 


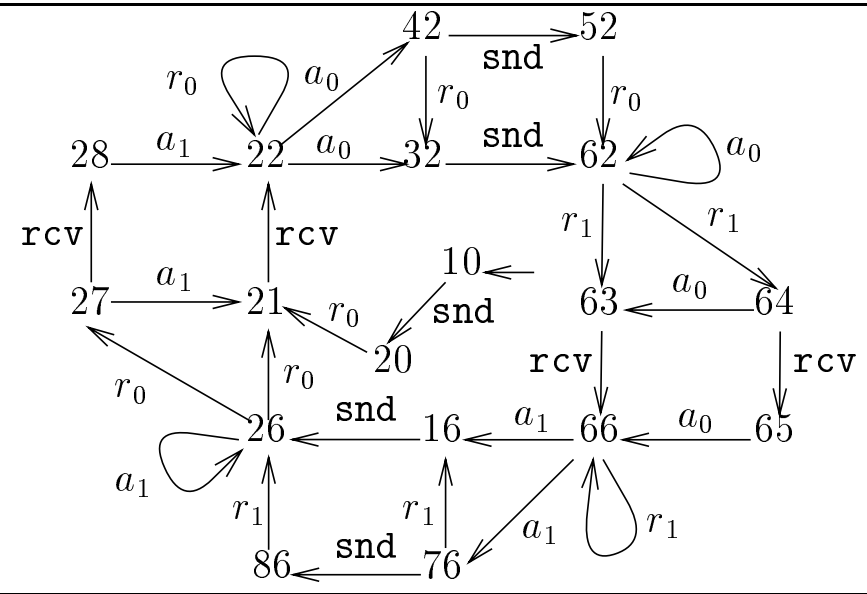

Figure 9 The reduced implementation.

First, consider the channel $M s_{1}={ }_{\theta} C_{1}\left[M s_{1}\right]$, shown in Figure 10. This channel is perhaps more realistic than $M s$, since the decision to lose or to deliver a message is taken after (and not while) accepting it with $s_{0}$ or $s_{1}$. The implementation remains correct if we change to $M s_{1}$ : since $\theta(M s) \simeq_{\text {shd }}^{c} C_{1}[M s]$ in arbitrary environments due to Axiom (10), according to the recursive pre-congruence of $\sqsubseteq_{\text {shd }}^{c}$ (Proposition 5 )

$A B P$ with $\theta_{M s}^{M s_{1}} \simeq_{\text {shd }}^{c} A B P$ with $\theta_{M s}^{C_{1}[M s]} \simeq_{\text {shd }}^{c} A B P$ with $\theta \simeq_{\text {shd }}^{c} B u f$

and hence (again due to Proposition 8) $A B P$ with $\theta_{M s}^{M s_{1}} \simeq$ obs Buf. Hence, in proving correctness with respect to should-testing, we can reuse the existing proof to a large degree. This argument, which is an example of the compositionality principle discussed in Section 3.4, fails for observational congruence, since $M s \simeq \mathbf{o b s} M s_{1}$ is false. The compositionality argument also fails for $\simeq_{\mathbf{f m s}}$ : we do have $M s \simeq_{\mathbf{f m s}} M s_{1}$, but since $\simeq_{\mathbf{f m s}}$ is not a congruence for hiding, we cannot exchange $M s$ and $M s_{1}$ in the context of $A B P$.

As a further variation, consider the channel $M s_{2}={ }_{\theta} C_{2}\left[M s_{2}\right]$. This channel cannot lose a message autonomously, but if the next message arrives before the previous one was read, then it overwrites this previous message. Since $C_{2}[M s] \coprod_{\text {shd }}^{c} C_{1}[M s]$ for arbitrary process environments by Axiom (13), it follows by recursive pre-congruence that

$$
A B P \text { with } \theta_{M s}^{M s_{2}} \sqsubseteq_{\text {shd }}^{c} A B P \text { with } \theta_{M s}^{C_{2}[M s]} \sqsubseteq_{\text {shd }}^{c} A B P \text { with } \theta_{M s}^{C_{1}[M s]} \simeq_{\text {shd }}^{c} B u f .
$$

Again, Proposition 8 then implies $A B P$ with $\theta_{M s}^{M s_{2}} \simeq$ obs $B u f$. The same argument works for the channel $M s_{3}={ }_{\theta} C_{3}\left[M s_{3}\right]$ used by Natarajan and Cleaveland (1995), where the part $\tau ; M s_{3}$ describes the autonomous decision to lose the message, which can also serve for freeing the channel for the next message.

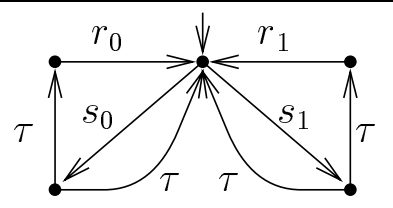

Figure 10 A different message channel $M s_{1}$. 


\section{CONCLUDING REMARKS}

In this paper we have attempted to demonstrate the practical usefulness of the fair testing preorder $\sqsubseteq_{\mathbf{s h d}}^{c}$ for the specification and analysis of distributed systems. To start, we have summarised in Sections 2 and 3 the main results achieved in Brinksma, Rensink and Vogler $(1995,1996)$. These results were mainly theoretical in nature, characterising $\complement_{\text {shd }}^{c}$ as the coarsest pre-congruence with respect to all usual process algebraic combinators, including hiding or abstraction as well as fixpoint-operators, contained in so-called fair must-testing (Brinksma (1988)). As such, they established $\sqsubseteq_{\text {shd }}^{c}$ as the answer to a long standing question, viz. whether it was possible to develop a testing pre-order that has fairness properties and is a pre-congruence with respect to abstraction.

In Section 4 we have presented a number of application examples to indicate also the practical usefulness of the new pre-order $\sqsubseteq_{\text {shd }}^{c}$. In Section 4.1 it is illustrated how external choice can be implemented by a busy-waiting loop traversing the initial states of the alternatives of the choice construct. This demonstrates that the expressiveness of $\Xi_{\text {shd }}^{c}$ is sufficiently greater than that of $\Xi_{\mathbf{f m s}}$, which cannot distinguish between productive and certain unproductive busy-waiting loops. Standard testing theories cannot deal with this example because the loops would cause catastrophic divergences.

In Section 4.2 we show the advantages of the fact that $\complement_{\text {shd }}^{c}$ is a congruence satisfying the proof principles presented in section 3, in a correctness proof for the well-known Alternating Bit-protocol. Comparable proofs in the literature have been carried out for observation congruence, see e.g. Larsen and Milner (1987), which satisfies fewer laws that can be used to reduce the complexity of the analysis. It is crucial here that $\square_{\text {shd }}^{c}$ is a pre-congruence in order for the proof to be carried out in the given, modular fashion.

This latter point is further exemplified by the extended analysis in Section 4.3, where a number of alternative communication channels are considered, each modelling a subtly different kind of data loss. Using the result of Section 4.2 and the fact that these descriptions are related through $\sqsubseteq_{\mathbf{s h d}}^{c}$, it can be readily concluded that the protocol is correct for each of these kinds of media. Again, observation congruence would fail a comparable modular proof strategy, because the different channels are not observation congruent, even if the protocol as a whole does implement the service modulo observation congruence. This coincides with the argument made by Valmari (1995) in favour of failure-based relations.

It is interesting to observe that these examples were all dealt with in the absence of the Recursive Specification Principle (RSP). RSP, which states that solutions of a set of guarded recursive equations are all congruent, does not hold for $\sqsubseteq_{\mathbf{s h d}}^{c}$ (see Brinksma, Rensink and Vogler (1995)). RSP is generally seen as a very desirable ingredient in the proof theory of process-algebraic congruences. It seems, however, that the fairness principle DFAR, together with the conditional reduction of $\sqsubseteq_{\text {shd }}^{c}$ to observation congruence of Proposition 8, suffices in many cases.

It is not yet completely understood whether the contraction lemma (Lemma 7), which is currently given in operational terms, has a proper algebraic formulation. So far, we have only found algebraic statements corresponding to special cases. This question is, of course, related to that of the complete axiomatisation of $\sqsubseteq_{\text {shd }}^{c}$, which is an important open question. We have the impression that if it exists at all, a complete axiomatisation will be very difficult to construct. 


\section{REFERENCES}

Baeten, J.C.M. and Weijland, W.P. (1990) Process Algebra, Cambridge University Press. Bolognesi, T. and Brinksma, E. (1987) Introduction to the ISO specification language LOTOS, Computer Networks and ISDN Systems, 14, 25-59.

Brinksma, E. (1988) A theory for the derivation of tests, in Protocol Specification, Testing, and Verification VIII (eds. S. Aggarwal and K. Sabnani), pp. 63-74, Elsevier Science Pub. B.V.

Brinksma, E. (1995) Cache consistency by design, in Protocol Specification, Testing, and Verification, XIV (eds. S. Vuong and S. Chanson), pp. 53-67, IFIP Series, Chapman \& Hall.

Brinksma, E., Rensink, A. and Vogler, W. (1995) Fair testing, in Concur '95: Concurrency Theory (eds. I. Lee and S. A. Smolka), pp. 313-27, vol. 962 of LNCS, Springer-Verlag.

Brinksma, E., Rensink, A. and Vogler, W. (1996) Fair testing, Hildesheimer InformatikBericht, Institut für Informatik, University of Hildesheim, in preparation.

Brookes, S.D., Hoare, C.A.R. and Roscoe, A.W. (1984) A theory of communicating sequential processes, J. ACM, 31(3), 560-99.

De Nicola, R. and Hennessy, M.C.B. (1984) Testing equivalences for processes, Theoretical Comput. Sci., 34, 83-133.

Glabbeek, R.J. van (1993) The linear time - branching time spectrum II: The semantics of sequential systems with silent moves, in Concur '93 (ed. E. Best), pp. 66-81, vol. 715 of $L N C S$, Springer-Verlag.

Groote, J.F. (1988) Implementation of events in LOTOS-specifications, Master's thesis, University of Twente.

Hennessy, M.C.B. (1988) Algebraic Theory of Processes, Foundations of Computing Series, MIT Press, Boston.

Hoare, C.A.R. (1985) Communicating Sequential Processes, Prentice-Hall.

ISO (1989) Information processing systems - open systems interconnection - LOTOS - a formal description technique based on the temporal ordering of observational behaviour, International Standard 8807, ISO, Geneva, 1st Edition.

Larsen, K.G. and Milner, R. (1987) Verifying a protocol using relativized bisimulation, in Automata, Languages and Programming (ed. T. Ottman), pp. 126-35, vol. 267 of $L N C S$, Springer-Verlag.

Milner, R. (1989) Communication and Concurrency, Prentice-Hall.

Natarajan, V. and Cleaveland, R. (1995) Divergence and fair testing, in Automata, Languages and Programming (eds. Z. Fülöp and F. Gécseg), vol. 944 of LNCS, SpringerVerlag.

Park, D. (1981) Concurrency and automata on infinite sequences, in Proceedings 5th GI Conference (ed. P. Deussen), pp. 167-83, vol. 104 of LNCS, Springer-Verlag.

Valmari, A. (1995) Failure-based equivalences are faster than many believe, in Structures in Concurrency Theory (ed. J. Desel), pp. 326-40, Workshops in Computing, SpringerVerlag.

Vogler, W. (1992) Modular Construction and Partial Order Semantics of Petri Nets, vol. 625 of $L N C S$, Springer-Verlag. 